\title{
The origin of biological information and programmed protein synthesis
}

Dan Liu

John Curtin School of Medical Research, Australian National University, Acton, Australia

Email: dan.liu@anu.edu.au

Received 22 July 2013; revised 15 August 2013; accepted 2 September 2013

Copyright (C) 2013 Dan Liu. This is an open access article distributed under the Creative Commons Attribution License, which permits unrestricted use, distribution, and reproduction in any medium, provided the original work is properly cited.

\begin{abstract}
Biological information is one of the most important characteristics of life, and it enables life to evolve to higher complexity and adapt to the environment by mutation and natural selection. However, the origin of this information recording and retrieval system remains a mystery. To understand the origin of biological information will lead us to one step closer to understand the origin of life on earth. Biological information is encoded in DNA and translated into protein by the ribosome in all free living organisms. The information has to be translated into proteins to carry out its biological functions, so the evolution of the ribosome must be integrated with the development of biological information. In this article, I propose that the small ribosomal subunit evolved from a ribozyme that acted as an RNA helicase in the ancient RNA world, and the involvement of tRNAs and the large ribosomal subunit evolved to enhance the helicase activity and to overcome the higher energy requirement for high GC content RNA helices. This process could have developed as a primitive recording mechanism: since Watson-Crick base paring is a natural property of RNA, each time the proto-small ribosomal subunit came to a particular GC-rich helix, tRNA-like molecules and the proto-large ribosomal subunit would have to be engaged to generate the helicase activity, and consequently the same polypeptide would be synthesized as a by-product. Simple recorded messages then evolved into useful biological information through continuous mutation and natural selection. This hypothesis provides logical and incremental steps for the development of programmed protein synthesis. I also argue that the helicase activity is preserved in the modern ribosome and that from our knowledge of the ribosome, and we can deduce the possible mechanisms of the helicase activity.
\end{abstract}

Keywords: Ribosome; tRNA; Translation; Translocation; mRNA Helicase; Evolution; Origin of Biological Information

\section{INTRODUCTION}

Biological information is contained in all the genes and intergenic regions with signals for gene expression. There are two major types of genes: one encodes information that is expressed as functional RNA molecules such as rRNA, tRNA, and snRNA, and another encodes information for protein synthesis expressed as mRNA. The functional RNA genes are thought to be remnants of an ancient RNA world [1-5] that existed prior to DNA and proteins, and their functions are involved in RNA editing or protein synthesis. The origin of the protein coding genes is unknown, and it is the major focus of this communication. Protein is one of the most important basic building blocks for life, and proteins are synthesized by the ribosome according to genetic information encoded in the genes of all living organisms. Since a gene can only carry out its biological function after it is translated into protein by the ribosome, the understanding of the evolution, structure and function of the ribosome should lead to an understanding of the origin of genetic information.

The ribosome is a large ribonucleoprotein complex and consists of small and large subunits (in bacteria 30S and 50S subunits, respectively, Figure 1(a). Transfer RNA (tRNA) is one of the key substrates in protein synthesis, and has an L-shaped structure with an anticodon at the end of one (anticodon) arm and a specific amino acid is linked to the end of the other (acceptor) arm (Figure 1(b)). Within the small ribosomal subunit the anticodon interacts with a codon on the message RNA (mRNA) by Watson-Crick base pairing and this process is called tRNA selection or decoding. When the cognate tRNA is selected, it delivers a specific amino acid into the pepti- 


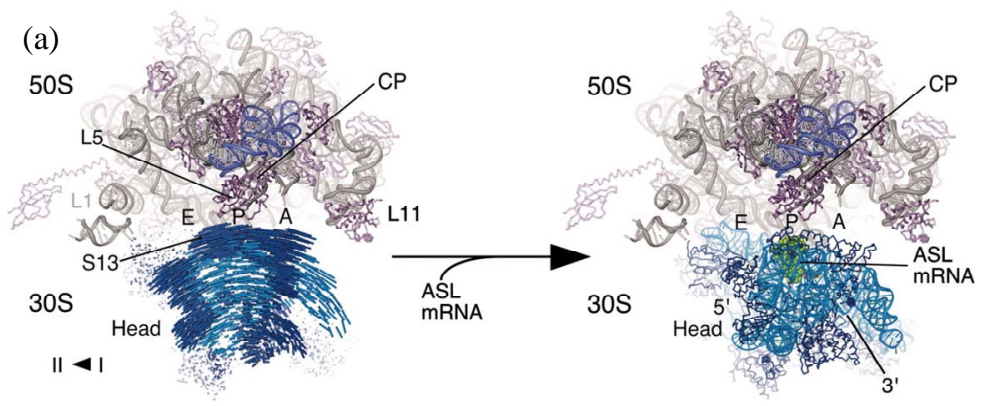

(b)

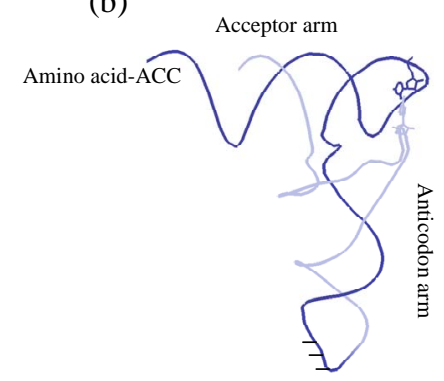

(c)
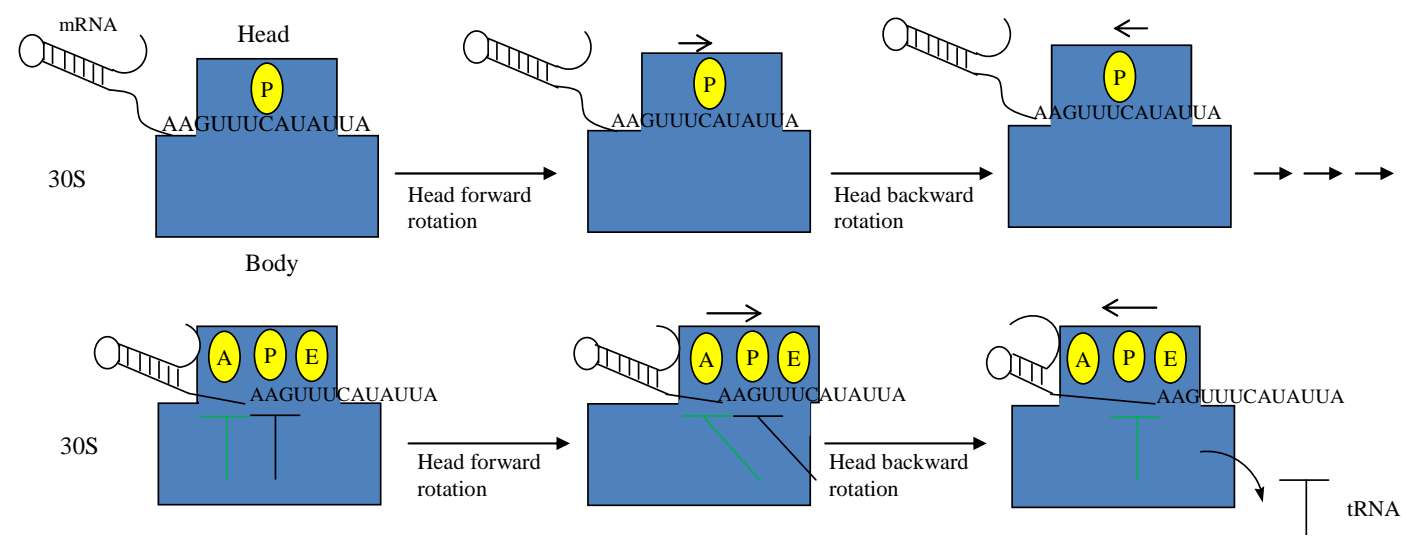

Figure 1. The structure of $E$. coli $70 \mathrm{~S}$ ribosome and the possible mRNA helicase function of the ribosome. (a) The structural rearrangement of the $70 \mathrm{~S}$ ribosome upon binding of mRNA and a P-site anticodon stem loop (ASL). Rearrangement of the 30S head position in the apo-70S ribosome structure are indicated by different vectors between phosphorous atoms (light blue) and $\mathrm{C} \alpha$ atom (dark blue). In the ribosome complex, the 5' to 3' direction of mRNA is indicated, and letters A, P and E represent the approximate positions of the tRNA binding sites at the subunit interface. Domains of the ribosome are labeled for the $30 \mathrm{~S}$ head (Head) and 50S central protuberance (CP), as are ribosomal protein in the small (S) and large (L) subunits (from Berk et al. [37], Copyright (2006) National Academy of Sciences, U. S. A.) (b) The yeast tRNA ${ }^{\text {Phe }}$ (PDB code 1EHZ) backbone structure is shown with the last three nucleotides of the tRNA (C74, C75, and A76) labeled. The first half (5') of the molecule is shown in light blue and the second (3') half in dark blue. (c) The two modes of helicase activity on the ribosome. Top panel (mode I) the 30S subunit could move along mRNA with minimal structure in the 5' to 3' direction based on the head movement of the 30S subunit. Lower panel (mode II) when the small subunit engages with a high GC-content helix, it could stall, and tRNAs could have a chance to bind to the mRNA and attract the large subunit. The movements of tRNAs in the ribosome could promote inter- and intra-subunit conformational changes, thereby enhancing helicase activity. The open arrows indicate the direction of the movement of the head domain and the large subunit and the acceptor part of tRNA are omitted in this illustration.

dyl transfer center (PTC) and a new peptidyl bond is formed in the large subunit (Figure 2). In this way the genetic information is converted into a specific amino acid sequence in a protein. There are three tRNA binding sites in both $30 \mathrm{~S}$ and $50 \mathrm{~S}$ ribosomal subunits (Figure 2): the A site for the incoming aminoacyl-tRNA, the P site for the peptidyl-tRNA, and the E site for the exiting deacylated tRNA from the ribosome. After peptide bond formation, the nascent peptide is transferred to the A site bound tRNA, and the P site bound tRNA is deacylated and spontaneously moves to the $\mathrm{E}$ site on the large subunit, while the anticodon still binds on the P site on the small subunit. This intermediate state with tRNAs bound to $\mathrm{A} / \mathrm{P}$ and $\mathrm{P} / \mathrm{E}$ positions is called the hybrid state (Figure 2) $[6,7]$. An elongation factor $G(E F-G)$ catalyzes the translocation of the mRNA/tRNA complex, and this process moves both tRNAs from the A and $\mathrm{P}$ sites to the $\mathrm{P}$ and $\mathrm{E}$ sites, respectively, and the mRNA advances by one codon (see in reviews [8,9]). During protein synthesis, the tRNA-mRNA complex is translocated through the ribosome along a path of more then $100 \AA$, and the translocation process involves a series of coordinated conformational changes affecting both subunits.

Numerous attempts have been made to describe the evolution of translation and the ribosome, such as Crick [1], Woese [10], Noller [11], Poole [12], Fox and Naik [13], and Wolf and Koonin [5] just to name a few. It has been suggested that the two subunits evolved independently $[11,14,15]$. The evolution of the large subunit has been subjected to intense studies and the suggestion has been made that it possibly evolved earlier than the small subunit [13]. It was suggested that the large subunit 


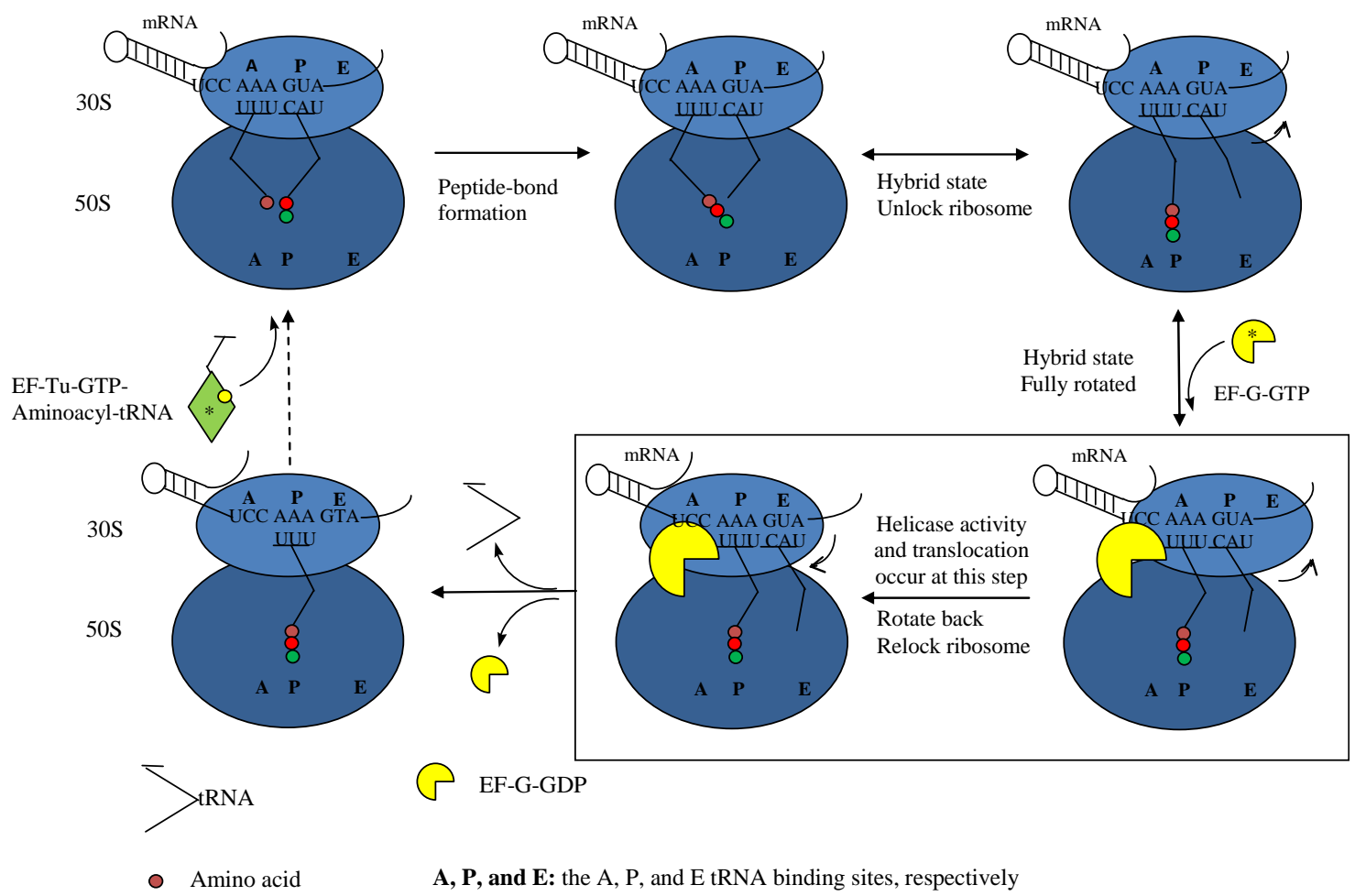

Figure 2. Elongation cycle of protein synthesis. The diagrams illustrate the inter-subunit rotation and the translocation of tRNAs from the A and P sites to the P and E sites, respectively. After peptide-bond formation, the acceptor ends of tRNAs spontaneously move from the A and P sites to the P and E sites, respectively, in the large ribosomal subunit forming the hybrid state. EFG-GTP binds to the ribosome and hydrolyzes GTP to GDP, and stabilizes the rotation of the small ribosomal subunit. At this step mRNA advances relative to the large subunit. When the small subunit rotates back, the translocation of mRNA/tRNA complex relative to the small subunit occurs and the returning head of the small subunit shears the mRNA helix at the entry of the downstream tunnel. This step is a novel suggestion in this communication and is framed in a box. The open arrows indicate the direction of the movement of the 30S subunit and its head domain.

could have evolved from a small RNA molecule of about 80 to110 nucleotides (nt) that could bind to tRNA-like small RNA molecules on their 3' CCA-amino acid ends. When a duplication event occurred, the resulting molecule could have formed two binding pockets side by side, which eventually developed into the A and P sites. It has been proposed that since two CCA-amino acid ends of RNA molecules could bind simultaneously in a very close proximity, it reduced the entropy and allowed peptide bond formation and gave rise to the primitive peptidyl transfer center (PTC) [13,16,17]. Recent studies [18, 19] seem to confirm this model. This reaction could have led to the formation of oligopeptides with random amino acid sequences. Random oligopeptides might offer higher degrees of complexity for RNA structures and capability for chemical catalysis $[3,20]$ and could consequently confer a huge advantage to the primitive "living" system. Hence, production of random peptides was selected. In order to improve the binding of the tRNA-like small RNAs (or proto-tRNA, pt-tRNA) and improve the efficiency of random oligopeptide synthesis driven by the selection, the proto-ribosome could have expanded in size and increased in complexity with evolutionary time. However, the further evolution of the capacity to synthesize a specific protein was still not possible since at this stage the peptide sequences were created by chance and there was no recording mechanism for repeatable peptide synthesis.

The origin of decoding must directly relate to the origin of biological information and programmed protein synthesis. However, it is hard to imagine how the decoding process or the coding system could have originated independently, as the codes would not have any meaning without a decoding system: a chicken-and-egg problem. Since evolution has no foresight, the code assignment and the decoding process had to evolve from other functions within the RNA world. A recent study [21] has suggested that the code assignment emerged before translation and could have been related to aminoacylating primordial tRNA by ribozymes mediated by the direct stereochemical affinity between amino acids and anticodons (or codons) [22] possibly for genomic 3' tag [14]. It has also been suggested that the small subunit possibly evolved from RNA polymerase [12,23]. In this model, 
pt-tRNAs would interact with an mRNA-like template (or proto-mRNA, pt-mRNA) in the proto-small subunit (pt-small subunit), and in a fashion similar to that of the codon and anticodon interaction. The "anticodon" was then cleaved from the pt-tRNA molecule and ligated with the nascent RNA. However, there is no evidence to suggest that the required nuclease or ligase activities ever existed on the ribosome. Furthermore that hypothesis does not provide a clear path that leads to programmed protein synthesis, nor an explanation for the association and coordinated actions of the small and large subunits. Due to the redundancy of codon recognition, this type of polymerization would have a mutation rate up to $30 \%$ that is much greater than the minimum replication fidelity $(<1 \%)$ required to conserve genetic information $[24$, 25]. It was also suggested [12] that the pt-mRNA could have served as an anchor for the pt-tRNAs to stabilize them on the PTC, thereby increasing the efficiency of the reaction. The pt-small subunit would have evolved the capacity to move the anchoring RNA in order to move the pt-tRNA directionally out of the PTC after the reaction. This hypothesis suggested that the anchoring RNA evolved into information carrying molecule for protein synthesis in the process $[5,12,26]$. However, this seems unlikely since the available experimental data have shown that the deacylated tRNA moves out of the PTC before the translocation of the mRNA [7].

In this communication, I put forward an alternative hypothesis that the small ribosomal subunit evolved from an RNA helicase, and suggest that this function has been preserved in the modern ribosome. In the RNA world, we assume that the RNA had template-dependent replication, but this would present a problem: how did the doublestranded RNA separate? If the strands did not separate, they could not form templates again or be folded into a ribozyme and there would be no replication cycle. Possibly at a very early stage, a geothermal pool could provide the energy for strand separation and the molecules could have circulated through the thermal gradient for their replication cycles. However, when the pool cooled down with geological changes over time, a helicase made from RNA and for RNA would become critical and would be as equally important as a polymerase under such conditions. I propose that the pt-small subunit could have been such a helicase. Furthermore pt-tRNA molecules could have enhanced the helicase activity, especially for the higher energy requirement of a GC-rich helix, by increasing the grip of the pt-small subunit on the pt-mRNA and by engaging the proto-ribosome (the large subunit). These steps simultaneously produce a polypeptide as a by-product. Since Watson-Crick base pairing is a natural property of RNA, each time the pt-small subunit came to the same GC-rich helix, the same polypeptide would have been synthesized; in essence, the first oligonucleo- tide reading mechanism linked to repeatable peptide formation. At the very beginning, this recording system would not have recorded any meaningful messages, but through mutation and natural selection, messages associated with useful peptides were continuously enhanced and conserved, giving rise to this type of biological information. This hypothesis is supported by the notion that the tRNA aminoacylated with earliest amino acids, such as glycine, alanine, valine and glutamic acid, which could be synthesized abioticly under primitive earth conditions [27], generally have the highest GC content in their anticodon and have the most stable interaction with the mRNA through Watson-Crick base pairing [28], and this could be essential for the proposed helicase activity. In fact, the coding regions in general have higher $\mathrm{G}+\mathrm{C}$ content compared to the non-coding regions even in modern genes [29,30], which might be the traceable mark of this process. A similar idea has been proposed by Zenkin in 2012 [31], however, no possible mechanism of the mRNA helicase activity related to the modern ribosome was given.

\section{THE EVIDENCE FOR THE RIBOSOME AS AN mRNA HELICASE}

The mechanism proposed here can only work if the ribosome has evolved from an mRNA helicase. Does any trace of this activity remain on the modern ribosome? The answer is affirmative. Noller and his colleagues have shown that the ribosome itself is an mRNA helicase [32]. In a purified system that only contained E. coli ribosomes, mRNA, tRNAs, and elongation factors EF-Tu and EF-G, the ribosome was able to disrupt stably basepaired RNA helixes, and the helicase active site was located in the mRNA entry tunnel (downstream tunnel) at a position +11 nt from the $\mathrm{P}$ site. In addition, helicase activity has also been shown to occur in a system that had only ribosomes, aa-tRNAs and the antibiotic sparsomycin [32] that has previously been shown to promote translocation without EF-G $[6,33]$. This indicates that the helicase activity may be directly linked to translocation, or that translocation is a part of the helicase activity. The coupling of helicase activity and translocation provides an important clue to explain how the coding and decoding system could have started, and shifts our fixed gaze on protein synthesis as the sole function of the ribosome to other possible functions.

A previous study using individual ribosomes and "optical tweezer" techniques demonstrated that the ribosome itself can unwind mRNA helices without an additional mRNA helicase [34]. They suggested that translocation and RNA unwinding are strictly coupled with ribosome functions. A recent study indicated that the ribosome as an mRNA helicase has two active mechanisms [35]. In 
the first mechanism, the mRNA helical junction was destabilized at the entry site on the ribosome by the interactions of the positively charged residues of the ribosomal proteins (r-protein) S3 and S4 and the backbone of mRNA at the helical junction. Mutation of these residues reduced the helicase activity, but did not eliminate it [32]. In the second mechanism, the mRNA helix is mechanically separated at the close junction during the inter- and intra-subunit ribosomal conformational changes and this was coupled with translocation. Qu and colleagues suggested that the conformational changes "generate a force that pulls on the tRNA: mRNA complex and promotes unwinding at the mRNA entry site”. This mechanism appears to be a unique property of the ribosomal mRNA helicase activity [35]. They also found that the translation rate is greatly influenced by the $\mathrm{G}+\mathrm{C}$ content of folded structures in the mRNA at the ribosomal entry site.

These findings indicate that the modern ribosome is still an mRNA helicase, and that the helicase activity is directly linked to the inter- and intra-subunit conformational changes during an elongation cycle. Therefore it is possible that this activity could have existed from the beginning and could have been the primordial function of the ancient ribosome before it acquired its function as a programmed protein synthesis machine.

\section{DECODING RESULTS FROM HELICASE ACTIVITY}

If the ribosome was and is an mRNA helicase, we should be able to explain all of its functions in terms of helicase activity. So, why is the decoding process (the interaction of tRNA and mRNA through Watson-Crick base paring) important for helicase activity? The proper base pairing of tRNA to mRNA increases the binding strength between the small subunit and the tRNA/mRNA complex on the A site [36] and the P site [37]. This increases the grip of the small subunit on mRNA. A mechanical study [38] demonstrated that addition of an aa-tRNA analog (N-acetylated Phe-tRNA ${ }^{\text {phe }}$ ) to an mRNA/ribosome complex strengthened the mRNA-ribosome bond.

In the current hypothesis, I suggest that the addition of tRNA to the small subunit could attract the large subunit and start the elongation cycle and the movements of tRNAs in the ribosome promoting inter- and intra-subunit conformational changes and therefore enhance the helicase activity (Figure 1(c), lower panel). As previously shown [7], after peptidyl transfer, the acceptor end of the deacylated tRNA travels spontaneously from the P site to the $\mathrm{E}$ site in the large subunit and the tRNA binds between the P/E positions (Figure 2). As a result of this movement the L shaped tRNA acts like a spring with one end bound to mRNA through the Watson-Crick base pairing and the $\mathrm{P}$ site of the small subunit and the other end interacts with the E site of the large subunits. The distance between the $\mathrm{P}$ site and the $\mathrm{E}$ site in the large subunit is about $50 \AA$ [39,40], and the change in position of tRNA from $\mathrm{P} / \mathrm{P}$ to $\mathrm{P} / \mathrm{E}$ creates a pulling force on the small subunit, that strongly favors the rotated position of the small subunit relative to the large subunit and the rotation of the head of the small subunit. These actions unlock the ribosome and are known to be important for translocation [41], and are also associated with helicase activity [35]. Improper base pairing of tRNA with mRNA would weaken the mRNA/tRNA complex, affect translocation, and cause frameshift mutations [42,43]. If there is no codon/anticodon interaction on the $\mathrm{P}$ site of the small subunit, the tRNA could not properly engage with the head of the small subunit and could not deliver the pull from the E site of the large subunit to the mRNA and the small subunit. Hence the interactions between the mRNA and the small subunit could not be dislodged and the translocation of mRNA would not occur. Consequently, with codon/anticodon base pairing, tRNAs act like a "handle" pulling mRNA forward, and this is why decoding is important for helicase activity and how the decoding mechanism could have been established before the coding system even existed.

\section{POSSIBLE HELICASE MECHANISM}

\subsection{Helicase Mechanism Mode I}

The ribosome has two possible modes of action as an mRNA helicase. The first mode involves the action of the small subunit without the involvement of tRNAs and the large subunit. It is well established that the head of the small subunit is inherently dynamic in the absence of binding of an anticodon stem loop (ASL) or a tRNA (Figure 1(a), [37,44]). I propose that this characteristic of the small subunit could mediate its helicase activity and the small subunit could have been evolved from an intrinsic RNA helicase ribozyme. It is likely that the pt-mRNA bound to the $\mathrm{P}$ site of the pt-small subunit in a sequence-independent manner through the phosphoribose backbone. When the head rotated towards the E site, the binding was no longer favorable in this position and the pt-mRNA would have been released (Figure 1(c), upper panel). When the head rotated back, a new interaction could form again. The disruption of RNA basepairing possibly occurred during the head domain rotation, or when the head rotates back away from the $\mathrm{E}$ site. Each cycle of translocation might have only disrupted one base-pair (Figure 1(c), upper panel), since there would be no reading frame.

This helicase activity is probably conserved in the modern ribosome and may relate to the activity called the ribosome scanning, when an mRNA is translocating from 
5' to 3' without the engagement of tRNAs. The scanning activity of the ribosome can be observed at the initiation of eukaryotic protein synthesis $[45,46]$ or in mRNA with translational bypass $[47,48]$, and scanning is undertaken by the small subunit alone [46] or the 70S ribosome [47]. A mechanical study of single ribosome/mRNA complexes [38] showed that there were three distinct groups of ribosome/mRNA complexes which were grouped according to rupture force (needed to pull the mRNA out of ribosome) less than $6 \mathrm{pN}$, between 6 and $15 \mathrm{pN}$, and between 15 and $25 \mathrm{pN}$. It is possible to speculate that these three groups represent the three different binding states of mRNA on the ribosome: releasing, head rotating and binding on the $\mathrm{P}$ site, respectively.

\subsection{Helicase Mechanism Mode II}

However, the proposed helicase activity of the (pt-)small subunit alone could only work on pt-mRNAs that had minimum secondary structure with a low $\mathrm{G}+\mathrm{C}$ content, as it would stall when it came to high $\mathrm{G}+\mathrm{C}$ helices due to their higher energy requirement. The proposed second mode of helicase activity evolved to bind the pt-aatRNAs to the pt-mRNA/small subunit complex and promote its engagement with a proto-ribosome, and this would have provided the extra energy for the helicase activity to act on high $\mathrm{G}+\mathrm{C}$ content mRNA helices as discussed above (Figure 1(c), lower panel). After peptide transfer, the hybrid state of tRNAs could pull the head of the small subunit towards the $\mathrm{E}$ site. When the movement is far enough and sustained long enough to release the binding of the $\mathrm{A}$ and $\mathrm{P}$ sites of the small subunit to the mRNA and the tRNAs, the spontaneous translocation would occur. However, in an extant system this rarely happens due to other forces acting on the mRNA/tRNAs complex, such as the bending of h44, and the binding of proteins S12 and S13 [37,49,50] that resist the rotation of the head of the small subunit and pull the tRNAs in the opposite direction back to the $\mathrm{A} / \mathrm{A}$ and $\mathrm{P} / \mathrm{P}$ positions. In support of this, ribosomes depleted of ribosomal proteins S12 and/or S13 increased spontaneous translocation [51], indicating that the force pulling the head of the small subunit towards the A site was reduced and thermodynamically favored the rotated state and therefore increasing spontaneous translocation. When ribosomes were treated with the thiol-specific agent $p \mathrm{CMB}$ ( $p$-chloromercuribenzoate), spontaneous translocation occurred and in some cases it could continue for greater then 40 elongation cycles [52]. The $p$-CMB agent targets the cysteine residues on the ribosomal proteins, indicating that the r-proteins regulate inter- and intra-subunit movements, and the regulatory action makes the movements more precise and efficient and that is important for programmed protein synthesis. However, the initiation of the movements and the movements themselves are most likely induced by the interactions between tRNAs and rRNAs.

If translocation was an inherent process of a helicase, in the very early stage in the development of the ribosome, it would not have had a strict reading frame. It is likely that each step of translocation could disrupt mostly 3 base pairs of an RNA helix, but could be 2 or 4 base pairs, depending on how far the small subunit's head could turn and how stable the RNA helices were; this would not have impaired the function of a helicase. Only when its by-products (proteins) were favorably selected for their much higher catalytic ability and functional versatility did a more measured translocation become important in order to establish a reading frame to reproduce the same protein each time. This step was subsequently regulated by evolving proteins such as elongation factor $\mathrm{G}$ (EF-G) and other r-proteins. This process would allow the development of programmed protein synthesis by incremental steps.

\section{HELICASE ACTIVITY OCCURS AT THE SECOND STEP OF TRANSLOCATION}

It has been well established by the means of toeprinting and the labeling of mRNA with pyrene [53-55] that when the small subunit and its head are in the rotated position, the mRNA/tRNA complex is not yet translocated. This indicates that the action of rotation does not in itself pull the mRNA into the entry tunnel, and means that the position of mRNA relative to the small subunit is unchanged. However, relative to the large subunit, the mRNA/tRNA complex is advanced by the rotation by at least $6 \AA$ [56, 57]. A recent study showed conclusively that the mRNA translocation occurred at the second step of inter-subunit rotation [58]. The first step of translocation is the counterclockwise rotation of the small subunit relative to the large subunit that happens rapidly with or without EF-G $[59,60]$, after the P site tRNA moves to the hybrid state conformation. The mechanism of this movement is not yet known, but it was suggested that the distorted shape of the $\mathrm{P}$ site tRNA might be the driving force for its movement from the $\mathrm{P} / \mathrm{P}$ to $\mathrm{P} / \mathrm{E}$ position [50]. The anticodon arm of the P site tRNA is deformed by the opposing interaction with the head of the small subunit and helix 69 of the large subunit; this results the opening of the major groove at the 26:44 base pair of the anticodon arm [50]. When the binding affinity between tRNA and the $\mathrm{P}$ site of the large subunit is reduced after deacylation, tRNA moves towards the E site to recover its more relaxed structure. Once the tRNAs move to the hybrid state, it unlocks the ribosome and inter-subunit rotation can take place. The second step of translocation is the clock- 
wise rotation that slowly restores the small subunit and its head back to the non-rotated state, and this coincided with the translocation of mRNA [58]. Furthermore, they demonstrated that the second step does not require EF-G release or GTP hydrolysis. It is possible that when the contacts between the mRNA/tRNA complex and the small subunit are finally broken, the torsion from the twisted head of the small subunit and the inertia of the reverse movement provide the disrupting force for the helicase activity and the translocation. The mRNA entry tunnel is very narrow at about $15 \AA$ and only allows a single strand of RNA to enter [61]. The returning head shears the RNA helix on the entrance of the tunnel, and pulls the mRNA into the tunnel, so the helicase activity and the translocation happen all at once (Figure 2 boxed). On the modern ribosome, the activities are enhanced by S3 and S4 proteins [32]. While the head of the small subunit is turning back, the mRNA is stabilized solely by the codon-anticodon interaction with tRNAs on the P site and $\mathrm{E}$ site. The tRNAs are in turn stabilized by the binding to the P site and the E site, and the L1 stalk on the large subunit, and EF-G + GDP which leans against the P site peptidyl-tRNA on the small subunit (Figure 2) [62, 63]. There will be 4 to 6 bases of codon-anticodon interaction due to the redundancy and only 0 to 3 base-pairing will need to be disrupted depending on the structure of mRNA for each step of translocation, indicating that this process is thermodynamically feasible. The current model implies that there is an energy barrier (disrupting an mRNA helix) to overcome when the head of the small subunit rotates back, and this process would put a strain on mRNA/tRNA complex. Consideration of this model provides a molecular explanation and demonstrates why the codon/anticodon interactions in the $\mathrm{P}$ and/or $\mathrm{E}$ sites are crucial for maintaining the reading frame. When there are defects in codon-anticodon interaction on the $\mathrm{P}$ site and/or the E site, it weakens the mRNA/tRNA complex, and when the head of the small subunit is turning towards the A site (the second step of translocation) and disrupting the mRNA helix, the pull would dislodge the mRNA from the tRNAs and cause frame shifts. This was observed by Zaher and Green [43] in a recent study. In some cases of programmed frameshifting, the pseudoknot on the mRNA, which interacts with the ribosome at the entrance of the downstream tunnel, presented a higher energy barrier for the helicase activity and translocation. The returning head of the small subunit pushed on the pseudoknot, and this dislodges mRNA from the tRNAs and causes frame shifting [64]. Cryo-EM studies on ribosomal intermediates stalled by a pseudoknot revealed the strain on the mRNA, the bending of the tip of h44 towards the A site, and distortion of the P site tRNA [64]. This observation supports the current model. At the other extreme, when the mRNA is devoid of secondary structure, such as poly- $U$, the codon-anticodon interaction was enough to stabilize mRNA without EF-G during translocation [65-67]. On the other hand, translocation was severely inhibited even in the presence of EF-G + GTP when the binding of tRNA in the P/E position was affected by the introduction of mutations in the $\mathrm{E}$ site of the large subunit $[68,69]$, or on the CCA end of the P/E bound tRNA [70-72], or in the absence of L1 protein [73]. This evidence indicates that tRNA is not only an adaptor for protein synthesis, but also plays an essential role in translocation by promoting ribosomal conformational changes and this is in the heart of helicase activity.

To summarize the possible of mechanism of helicase activity and translocation: the hybrid state of tRNAs strongly favors the rotation of the small subunit relative to the large subunit and the rotation of its head towards the E site. This movement partially advances the mRNA relative to the large subunit. The rotation weakens the binding between the small subunit and the mRNA/tRNA complex [74], and eventually when the complex is released the tRNAs adopt the $\mathrm{P}$ and $\mathrm{E}$ site positions because of the binding of the large subunits to the tRNAs on the $\mathrm{P}$ and $\mathrm{E}$ sites, respectively. At this stage, the mRNA is completely translocated relative to the large subunit, but the small subunit and its head are still rotating back. When the rotations are completed, the translocation relative to the small subunit and helicase activity are accomplished (Figure 2). In this process, most of the contacts and movements are between rRNAs and tRNAs, so one could envisage that the similar action could happen in an all RNA ribosome; albeit it might not be very precise.

\section{GENERATION OF CODING INFORMATION}

The origins of genetic information and programmed protein synthesis have been an enduring enigma for biologists since the discovery of DNA and the ribosome in the 1950s. The debate between "genetic heredity-first" and "metabolism-first" still continues. The recent "protein centric" views of evolution of life [75-77] suggested that proteins evolved first before the ribosome, and that the peptides or proteins could be synthesized by other peptides or proteins in the system and this process was "autocatalytic". The hypothesis was based on phylogenetic studies of protein structures and RNA, and one of main arguments was that the proteins with the oldest folds were metabolic enzymes, instead of proteins related to translation or RNA binding. The major difficulties of this hypothesis are not only the lack of evidence for the "autocatalytic" process, but also it is without a viable mechanism to transfer the information of these proteins to RNA or DNA. All the information of these ancient 
proteins would be lost in the transition from the noncoding to the coding system and it is unlikely that the proteins reinvented by coding synthesis would be identical to their ancient counterparts, indicating that the methods used in their study cannot reach the point beyond translation.

The present hypothesis describes how genetic information could have been generated from the ancient RNA world through a recording system and natural selection. At the very early stage, the pt-small and pt-large subunits as ribozymes could have evolved separately with different functions, such as helicase and the production of random peptides, respectively. Template-dependent replication and folded RNA structures form double helices as a natural property of RNA, however these structures could prevent further replication. To facilitate the essential helicase activity, the two subunits combined with aminoacylated primordial tRNA could have cooperated and produced random peptides as by-product. Some of these peptides could have improved the fitness of the primitive "living" system, including the ones that could have basic metabolic function or ability to stabilize RNA molecules. The RNA molecules encoding these peptides would be selected and could form the basis for repeatable peptide production. Mutations on these RNA molecules could provide variants for natural selection and could lead to a better adaptation to the environment for early life. In this way, the genetic information could have formed and continued to improve and diversify, and pave the way for the transition from the RNA world to the RNA/protein world. The ribosome could also mature and become more efficient and have higher fidelity with the involvement of proteins in the same process. Unlike the previous models for the origin of the small ribosomal subunit mentioned in introduction, which are speculative and without experimental support, the present hypothesis is based on the natural property of RNA molecules, the principle of Darwinian evolution, and our current knowledge of the ribosome and provides a sensible and logical solution for the enduring enigma.

\section{CONCLUSIONS}

So far, I described the ribosome as an mRNA helicase with two modes of action and reviewed the evidence that the modern ribosome has the mRNA helicase function. I believe that the helicase function of the ribosome existed prior to and led to the emergence of programmed protein synthesis. tRNA plays a central role in function in helicase activity. In 1970, Woese proposed that mRNA was pulled through the ribosome by a tRNA ratchet [10]. Although the detail of the proposed mechanism might not be in agreement with our current knowledge of the ribosome, the basic idea was correct. Woese stated that "it seems impossible to avoid invoking tRNA-like entities (that is an 'adaptor' system) as an integral part of translation from its very inception-which is sufficient reason for suspecting the basic molecular mechanisms of the process to lie in the properties of this molecular species". The present model describes the early inception of the translation system and the role of tRNA based on the properties of tRNA and our current knowledge of the ribosome. This model also explains the necessity of the coordinated movements of the small and large subunits, and the possibility of the establishment of a recording mechanism. The biological information was developed from this recoding mechanism through continuous mutation and natural selection. The interaction of the small and large subunits could also improve the efficiency of peptide bond formation, since the substrates (tRNAs) are securely enveloped in the interface between the two subunits, so the present proposal does not contradict Fox's large subunit-first model. It seems that the ribosome is the only example of this type of RNA helicase existing in the modern world, and it is possible this helicase only evolved once in the history of evolution or other similar RNA helicases were replaced by the most successful pt-ribosome. It is still not clear which part of the small subunit is original. It has been suggested that the 3' domain of the 16S rRNA including h44 and the $\mathrm{P}$ site are the oldest parts of the small subunit [26,77-79], and this is consistent with the present proposal. The dynamic movement of the head of the small subunit is at the center of its helicase activity. Understanding the molecular basis that sustains this movement would lead to the understanding of evolution of the small subunit.

It is widely argued that the RNA world preceded the DNA and protein world. Today, although almost all the functions that were carried out by RNAs in the RNA world have been replaced by proteins, the function of the ribosome, which is essentially RNA machinery, has not. Proteins have not yet evolved the ability to synthesize themselves according to genetic information after more than three billion years of evolution. This gives us a unique opportunity to glance back to the lost RNA world through the structure and functions of the ribosome.

\section{ACKNOWLEDGEMENTS}

I thank Dr. David A. Bastin and Prof. Philip Board for their careful reading of the manuscript and very helpful discussions, and Dr. Huina Zhou for creating figures. I would like to thank Prof. Harry Noller for his work on the ribosome and translocation that makes this work possible.

\section{REFERENCES}

[1] Crick, F.R. (1968) The origin of genetic code. Journal of 
Molecular Biology, 38, 367-379. http://dx.doi.org/10.1016/0022-2836(68)90392-6

[2] Gilbert, W. (1986) The RNA world. Nature, 319, 618. http://dx.doi.org/10.1038/319618a0

[3] Noller, H. (2004) The driving force for molecular evolution of transloation. RNA, 10, 1833-1837. http://dx.doi.org/10.1261/rna.7142404

[4] Orgel, L.E. (1968) Evolution of the genetic apparatus. Journal of Molecular Biology, 38, 381-393. http://dx.doi.org/10.1016/0022-2836(68)90393-8

[5] Wolf, Y.I. and Koonin, E.V. (2007) On the origin of the translation system and genetic code in the RNA world by means of natural selection, exaptation, and subfunctionalisztion. Biology Direct, 2, 14-39. http://dx.doi.org/10.1186/1745-6150-2-14

[6] Dorner, S., Brunelle, J.L., Sharma, D. and Green, R. (2006) The hybrid state of tRNA binding is an authentic translation elongation intermediate. Nature Structural \& Molecular Biology, 13, 234-241. http://dx.doi.org/10.1038/nsmb1060

[7] Moazed, D. and Noller, H.F. (1989) Intermediate states in the movement of transfer RNA in the ribosome. Nature, 342, 142-148. http://dx.doi.org/10.1038/342142a0

[8] Joseph, S. (2003) After the ribosome structure: How does translocation work? RNA, 9, 160-164. http://dx.doi.org/10.1261/rna.2163103

[9] Korostelev, A., Ermolenko, D.N. and Noller, H.F. (2008) Structural dynamics of the ribosome. Current Opinion in Chemical Biology, 12, 674-683. http://dx.doi.org/10.1016/j.cbpa.2008.08.037

[10] Woese, C. (1970) Molecular mechanics of translation: A reciprocating ratchet mechanism. Nature, 226, 817-820. http://dx.doi.org/10.1038/226817a0

[11] Noller, H.F. (1993) On the origin of the ribosome: Coevolution of subdomains of tRNA and rRNA. In: Gesteland, R.F. and Atkins, J.F. Eds., The RNA world, Cold Spring Harbor Laboratory Press, Cold Spring Harbor, New York, 137-156.

[12] Poole, A.M., Jeffares, D.C. and Penny, D. (1998) The path from the RNA world. Journal of Molecular Evolution, 46, 1-17. http://dx.doi.org/10.1007/PL00006275

[13] Fox, G.E. and Naik, A.K. (2004) The evolutionary history of the translation machinery. In: De Pouplana, R.L. Ed., The Genetic Code and The Origin of Life, Landers BioScience, Georgetown, 92-105. http://dx.doi.org/10.1007/0-387-26887-1_6

[14] Maizels, N. and Weiner, A.M. (1993) The genomic tag hypothesis: Modern viruses as molecular fossils of ancient strategies for genomic replication. In: Gesteland, R.F. and Atkins, J.F., Eds., The RNA world, Cold Spring Harbor Laboratory Press, Cold Spring Harbor, New York, 577-602.

[15] Schimmel, P., Giege, R., Moras, D. and Yokoyama, S. (1993) An operational RNA code for amino acids and possible relationship to genetic code. Proceedings of the National Academy of Sciences of the United States of
America, 90, 8763-8768. http://dx.doi.org/10.1073/pnas.90.19.8763

[16] Agmon, I., Bashan, A. and Yonath, A. (2006) On ribosome conservation and evolution. Israel Journal of Ecology and Evolution, 52, 359-379. http://dx.doi.org/10.1560/IJEE_52_3-4_359

[17] Sievers, A., Beringer, M., Rodnina, M.V. and Wolfenden, R. (2004) The ribosome as an entropy trap. Proceedings of the National Academy of Sciences of the United States of America, 101, 7897-7901. http://dx.doi.org/10.1073/pnas.0402488101

[18] Bokov, K. and Steinberg, S.V. (2009) A hierarchical model for evolution of 23S ribosomal RNA. Nature, 457, 977-980. http://dx.doi.org/10.1038/nature07749

[19] Hsiao, C., Mohan, S., Kalahar, B.K. and Williams, L.D. (2009) Peeling the onion: ribosomes are ancient molecular fossils. Molecular Biology and Evolution, 26, 24152425. http://dx.doi.org/10.1093/molbev/msp163

[20] Mzathmary, E. and Smith, M. (1997) From replicators to re-producers: The first major transition leading to life. Journal of Theoretical Biology, 187, 555-571. http://dx.doi.org/10.1006/jtbi.1996.0389

[21] Rodin, A.S., Szathmary, E. and Rodin, S.N. (2011) On origin of genetic code and tRNA before translation. Biology Direct, 6, 14. http://dx.doi.org/10.1186/1745-6150-6-14

[22] Rodin, A.S., Szathmary, E. and Rodin, S.N. (2009) On ancestor for codes viewed from the perspective of two complementary modes of tRNA aminoacylation. Biology Direct, 4, 4. http://dx.doi.org/10.1186/1745-6150-4-4

[23] Gordon, K.H.J. (1995) Were RNA replication and translation directly coupled in the RNA (+protein?) world? Journal of Theoretical Biology, 173, 179-193. http://dx.doi.org/10.1006/jtbi.1995.0054

[24] Eigen, M. (1971) Selforganization of matter and the evolution of biological macromolecules. Die Naturwissenschaften, 58, 465-523.

http://dx.doi.org/10.1007/BF00623322

[25] Kun, A., Santos, M. and Szathmary, E. (2005) Real ribozymes suggest a relaxed error threshold. Nature Genetics, 37, 1008-1011. http://dx.doi.org/10.1038/ng1621

[26] Fox, G.E. (2010) Origin and evolution of the ribosome. Cold Spring Harbor Perspectives in Biology, 9, a003483. http://dx.doi.org/10.1101/cshperspect.a003483

[27] Miller, S.L. (1953) A production of amino acids under possible primitive earth conditions. Science, 117, 528529. http://dx.doi.org/10.1126/science.117.3046.528

[28] Travers, A. (2006) The evolution of the genetic code revisited. Origins of Life and Evolution of Biospheres, 36, 549-555. http://dx.doi.org/10.1007/s11084-006-9041-6

[29] Bultrini, E. and Pizzi, E. (2006) A new parameter to study compositional properties of non-coding regions in eukaryotic genomes. Gene, 385, 75-82. http://dx.doi.org/10.1016/j.gene.2006.05.030

[30] Grantham, R., Gautier, C., Gouy, M., Mercier, R. and Pave, A. (1980) Codon catalog usage and the genome hypothesis. Nucleic Acids Research, 8, r49-r62. 
http://dx.doi.org/10.1093/nar/8.1.197-c

[31] Zenkin, N. (2012) Hypothesis: Emergence of translation as a result of RNA helicase evolution. Journal of Molecular Evolution, 74, 249-256.

http://dx.doi.org/10.1007/s00239-012-9503-6

[32] Takyar, S., Hickerson, R.P. and Noller, H.F. (2005) mRNA helicase activity of the ribosome. Cell, 120, 49-58. http://dx.doi.org/10.1016/j.cell.2004.11.042

[33] Fredrick, K. and Noller, H.F. (2003) Catalysis of ribosomal translocation by sparsomycin. Science, 300, 11591162.http://dx.doi.org/10.1126/science.1084571

[34] Wen, J.D., Lancaster, L., Hodges, C., Zeri, A.C., Yoshimura, S.H. and Noller, H.F., et al. (2008) Following translation by single ribosomes one codon at a time. $\mathrm{Na}$ ture, 452, 598-603. http://dx.doi.org/10.1038/nature06716

[35] Qu, X., Wen, J.D., Lancaster, L., Noller, H.F., Bustamante, C. and Tinoco, I. Jr. (2011) The ribosome uses two active mechanisms to unwind messenger RNA during translation. Nature, 475, 118-121. http://dx.doi.org/10.1038/nature10126

[36] Ogle, J.M., Brodersen, D.E., Clemons Jr., W.M., Tarry, M.J., Carter, A.P. and Ramakrishnan, V. (2001) Recognition of cognate transfer RNA by the $30 \mathrm{~S}$ ribosomal subunit. Science, 292, 897-902. http://dx.doi.org/10.1126/science.1060612

[37] Berk, V., Zhang, W., Pai, R.D. and Cate, J.H. (2006) Structural basis for mRNA and tRNA positioning on the ribosome. Proceedings of the National Academy of Sciences of the United States of America, 103, 15830-15834. http://dx.doi.org/10.1073/pnas.0607541103

[38] Vanzi, F., Takagi, Y., Shuman, H., Cooperman, B.S. and Goldman, Y.E. (2005) Mechanical studies of single ribosome/mRNA complexes. Biophysical Journal, 89, 19091919. http://dx.doi.org/10.1529/biophysj.104.056283

[39] Korostelev, A., Trakhanov, S., Laurberg, M. and Noller, H.F. (2006) Crystal structure of a 70S ribosome-tRNA complex reveals functional interactions and rearrangements. Cell, 126, 1065-1077. http://dx.doi.org/10.1016/j.cell.2006.08.032

[40] Noller, H.F. and Baucom, A. (2002) Structure of the $70 \mathrm{~S}$ ribosome: Implications for movement. Biochemical Society Transactions, 30, 1159-1161. http://dx.doi.org/10.1042/BST0301159

[41] Horan, L.H. and Noller, H.F. (2007) Intersubunit movement is required for ribosomal translocation. Proceedings of the National Academy of Sciences of the United States of America, 104, 4881-4885. http://dx.doi.org/10.1073/pnas.0700762104

[42] Muldoon-Jacobs, K.L. and Dinman, J.D. (2006) Specific effects of ribosome-tethered molecular chaperones on programmed -1 ribosomal frameshifting. Eukaryotic Cell, 5, 762-770. http://dx.doi.org/10.1128/EC.5.4.762-770.2006

[43] Zaher, H.S. and Green, R. (2009) Quality control by the ribosome following peptide bond formation. Nature, 457, 161-166.

[44] Schuwirth, B.S., Borovinskaya, M.A., Hau, C.W., Zhang,
W., Vila-Sanjurjo, A., Holton, J.M., et al. (2005) Structures of the bacterial ribosome at 3.5 A resolution. Science, 310, 827-834. http://dx.doi.org/10.1126/science.1117230

[45] Kozak, M. (1978) How do eucaryotic ribosomes select initiation regions in messenger RNA? Cell, 15, 11091123. http://dx.doi.org/10.1016/0092-8674(78)90039-9

[46] Kozak, M. (1989) The scanning model for translation: An update. The Journal of Cell Biology, 108, 229-241. http://dx.doi.org/10.1083/jcb.108.2.229

[47] Herr, A.J., Atkins, J.F. and Gesteland, R.F. (1999) Mutations which alter the elbow region of tRNA2Gly reduce T4 gene 60 translational bypassing efficiency. The EMBO Journal, 18, 2886-2896. http://dx.doi.org/10.1093/emboj/18.10.2886

[48] Weiss, R.B., Huang, W.M. and Dunn, D.M. (1990) A nascent peptide is required for ribosomal bypass of the coding gap in bacteriophage T4 gene 60. Cell, 62, 117126. http://dx.doi.org/10.1016/0092-8674(90)90245-A

[49] Ogle, J.M., Murphy, F.V., Tarry, M.J. and Ramakrishnan, V. (2002) Selection of tRNA by the ribosome requires a transition from an open to a closed form. Cell, 111, 721732. http://dx.doi.org/10.1016/S0092-8674(02)01086-3

[50] Selmer, M., Dunham, C.M., Murphy, F.V., Weixlbaumer, A., Petry, S., Kelley, A.C., et al. (2006) Structure of the $70 \mathrm{~S}$ ribosome complexed with mRNA and tRNA. Science, 313, 1935-1942. http://dx.doi.org/10.1126/science.1131127

[51] Cukras, A.R., Southworth, D.R., Brunelle, J.L., Culver, G.M. and Green, R. (2003) Ribosomal proteins S12 and S13 function as control elements for translocation of the mRNA: tRNA complex. Molecular Cell, 12, 321-328. http://dx.doi.org/10.1016/S1097-2765(03)00275-2

[52] Southworth, D.R., Brunelle, J.L. and Green, R. (2002) EFG-independent translocation of the mRNA: tRNA complex is promoted by modification of the ribosome with thiol-specific reagents. Journal of Molecular Biology, 324, 611-623. http://dx.doi.org/10.1016/S0022-2836(02)01196-8

[53] Pan, D., Kirillov, S.V. and Cooperman, B.S. (2007) Kinetically competent intermediates in the translocation step of protein synthesis. Molecular Cell, 25, 519-529. http://dx.doi.org/10.1016/j.molcel.2007.01.014

[54] Spiegel, P.C., Ermolenko, D.N. and Noller, H.F. (2007) Elongation factor $\mathrm{G}$ stabilizes the hybrid-state conformation of the 70S ribosome. RNA, 13, 1473-1482. http://dx.doi.org/10.1261/rna.601507

[55] Zavialov, A.V., Hauryliuk, V.V. and Ehrenberg, M. (2005) Guanine-nucleotide exchange on ribosome-bound elongation factor $\mathrm{G}$ initiates the translocation of tRNAs. Journal of Biology, 4, 9. http://dx.doi.org/10.1186/jbiol24

[56] Agirrezabala, X., Lei, J., Brunelle, J.L., Ortiz-Meoz, R.F., Green, R. and Frank, J. (2008) Visualization of the hybrid state of tRNA binding promoted by spontaneous ratcheting of the ribosome. Molecular Cell, 32, 190-197. http://dx.doi.org/10.1016/j.molcel.2008.10.001

[57] Dunkle, J.A., Wang, L., Feldman, M.B., Pulk, A., Chen, V.B., Kapral, G.J., et al. (2011) Structures of the bacterial 
ribosome in classical and hybrid states of tRNA binding. Science, 332, 981-984. http://dx.doi.org/10.1126/science.1202692

[58] Ermolenko, D.N. and Noller, H.F. (2011) mRNA translocation occurs during the second step of ribosomal intersubunit rotation. Nature Structural \& Molecular Biology, 18, 457-462.

[59] Cornish, P.V., Ermolenko, D.N., Noller, H.F. and Ha, T. (2008) Spontaneous intersubunit rotation in single ribosomes. Molecular Cell, 30, 578-588.

http://dx.doi.org/10.1016/j.molcel.2008.05.004

[60] Fischer, N., Konevega, A.L., Wintermeyer, W., Rodnina, M.V. and Stark, H. (2010) Ribosome dynamics and tRNA movement by time-resolved electron cryomicroscopy. Nature, 466, 329-333.

[61] Borovinskaya, M.A., Shoji, S., Holton, J.M., Fredrick, K. and Cate, J.H.D. (2007) A steric block in translation caused by the antibiotic spectinomycin. ACS Chemical Biology, 2, 545-552. http://dx.doi.org/10.1021/cb700100n

[62] Frank, J. and Agrawal, R.K. (2001) Ratchet-like movements between the two ribosomal subunits: Their implications in elongation factor recognition and tRNA translocation. Cold Spring Harbor Symposia on Quantitative Biology, 66, 67-75. http://dx.doi.org/10.1101/sqb.2001.66.67

[63] Julian, P., Konevega, A.L., Scheres, S.H., Lazaro, M., Gil, D., Wintermeyer, W., et al. (2008) Structure of ratcheted ribosomes with tRNAs in hybrid states. Proceedings of the National Academy of Sciences of the United States of America, 105, 16924-16927. http://dx.doi.org/10.1073/pnas.0809587105

[64] Namy, O., Moran, S.J., Stuart, D.I., Gilbert, R.J. and Brierley, I. (2006) A mechanical explanation of RNA pseudoknot function in programmed ribosomal frameshifting. Nature, 441, 244-247.

[65] Gavrilova, L.P., Kostiashkina, O.E., Koteliansky, V.E., Rutkevitch, N.M. and Spirin, A.S. (1976) Factor-free ("non-enzymic") and factor-dependent systems of translation of polyuridylic acid by Escherichia coli ribosomes. Journal of Molecular Biology, 101, 537-552. http://dx.doi.org/10.1016/0022-2836(76)90243-6

[66] Gavrilova, L.P. and Spirin, A.S. (1971) Stimulation of "non-enzymic" translocation in ribosomes by p-chloromercuribenzoate. FEBS Letters, 17, 324-326. http://dx.doi.org/10.1016/0014-5793(71)80177-1

[67] Pestka, S. (1974) Assay for nonenzymatic and enzymatic translocation with Escherichia coli ribosomes. Methods in Enzymology, 30, 462-470. http://dx.doi.org/10.1016/0076-6879(74)30046-8

[68] Sergiev, P.V., Lesnyak, D.V., Kiparisov, S.V., Burakovsky, D.E., Leonov, A.A., Bogdanov, A.A., et al. (2005) Function of the ribosomal E-site: A mutagenesis study. Nucleic Acids Research, 33, 6048-6056. http://dx.doi.org/10.1093/nar/gki910
[69] Walker, S.E., Shoji, S., Pan, D., Cooperman, B.S. and Fredrick, K. (2008) Role of hybrid tRNA-binding states in ribosomal translocation. Proceedings of the National Academy of Sciences of the United States of America, 105, 9192-9197. http://dx.doi.org/10.1073/pnas.0710146105

[70] Feinberg, J.S. and Joseph, S. (2001) Identification of molecular interactions between P-site tRNA and the ribosome essential for translocation. Proceedings of the National Academy of Sciences of the United States of America, 98, 11120-11125. http://dx.doi.org/10.1073/pnas.211184098

[71] Lill, R., Robertson, J.M. and Wintermeyer, W. (1989) Binding of the 3' terminus of tRNA to 23S rRNA in the ribosomal exit site actively promotes translocation. The EMBO Journal, 8, 3933-3938.

[72] Virumae, K., Saarma, U., Horowitz, J. and Remme, J. (2002) Functional importance of the 3'-terminal adenosine of tRNA in ribosomal translation. The Journal of Biological Chemistry, 277, 24128-24134. http://dx.doi.org/10.1074/jbc.M200393200

[73] Subramanian, A.R. and Dabbs, E.R. (1980) Functional studies on ribosomes lacking protein L1 from mutant Escherichia coli. European Journal of Biochemistry, 112, 425-430. http://dx.doi.org/10.1111/j.1432-1033.1980.tb07222.x

[74] Uemura, S., Dorywalska, M., Lee, T.H., Kim, H.D., Puglisi, J.D. and Chu, S. (2007) Peptide bond formation destabilizes shine-dalgarno interaction on the ribosome. Nature, 446, 454-457.

[75] Caetano-Anolles, D., Kim, K.M., Mittenthal, J.E. and Caetano-Anolles, G. (2011) Proteome evolution and the metabolic origins of translation and cellular life. Journal of Molecular Evolution, 72, 14-33. http://dx.doi.org/10.1007/s00239-010-9400-9

[76] Caetano-Anolles, G., Kim, K.M. and Caetano-Anolles, D. (2012) The phylogenomic roots of modern biochemistry: Origins of proteins, cofactors and protein biosynthesis. Journal of Molecular Evolution, 74, 1-34. http://dx.doi.org/10.1007/s00239-011-9480-1

[77] Harish, A. and Caetano-Anolles, G. (2012) Ribosomal history reveals origins of modern protein synthesis. PloS One, 7, e32776. http://dx.doi.org/10.1371/journal.pone.0032776

[78] Bernhardt, H.S. and Tate, W.P. (2010) The transition from noncoded to coded protein synthesis: Did coding mRNAs arise from stability-enhancing binding partners to tRNA? Biology Direct, 5, 16-29. http://dx.doi.org/10.1186/1745-6150-5-16

[79] Schafer, M.A., Tastan, A.O., Patzke, S., Blaha, G., Spahn, C.M., Wilson, D.N., et al. (2002) Codon-anticodon interaction at the $\mathrm{P}$ site is a prerequisite for tRNA interaction with the small ribosomal subunit. The Journal of Biological Chemistry, 277, 19095-19105. http://dx.doi.org/10.1074/jbc.M108902200 